\title{
AVALIAÇÃO DA QUALIDADE DAS MEDIDAS EXPERIMENTAIS NAS REGIÕES DE CONFIANÇA DOS PARÂMETROS CINÉTICOS ESTIMADOS DE UM SISTEMA COM REAÇÕES EM SÉRIE
}

\author{
H. ENZWEILER ${ }^{1}$, E. B. COUTINHO ${ }^{2}$ e M. SCHWAAB ${ }^{3}$ \\ ${ }^{1}$ Universidade Estadual de Maringá, Departamento de Engenharia Química \\ ${ }^{2}$ Universidade Federal do Rio Grande do Sul, Departamento de Físico-Química \\ ${ }^{3}$ Universidade Federal Santa Maria, Departamento de Engenharia Química \\ E-mail para contato: marcioschwaab@gmail.com
}

\begin{abstract}
RESUMO - Em sistemas compostos por diversas reações em série e/ou paralelo nem sempre há a possibilidade de medir-se a concentração de todas as espécies envolvidas ou, ainda, as medidas destas variáveis não são totalmente independentes. Nestes casos, a qualidade dos valores estimados para os parâmetros cinéticos do sistema depende do número de componentes medidos e do grau de correlação entre as medições. Para um sistema reacional em série do tipo $\mathrm{A} \rightarrow \mathrm{B} \rightarrow \mathrm{C}$ foi avaliado o efeito da quantidade de medidas experimentais e da presença de correlação entre as medidas experimentais sobre a região de confiança dos parâmetros estimados. Observou-se que apenas com as medidas de concentração do produto final obtém-se uma região de confiança extensa e com forma distante da elíptica. Foi verificado que o valor da correlação modifica significativamente o tamanho e a forma da região de confiança dos parâmetros cinéticos, podendo ainda causar o seu deslocamento para intervalos de valores diferentes.
\end{abstract}

\section{INTRODUÇÃO}

No caso de sistemas que envolvem reações em série ou em paralelo, a determinação de parâmetros cinéticos geralmente envolve a utilização de medidas da concentração dos compostos envolvidos na reação. Contudo, nem sempre há a possibilidade de obter as medidas para todos os compostos envolvidos (Box e Draper, 1965), ou mesmo estas medidas não podem ser obtidas de maneira independente, sem correlação (Santos e Pinto, 1998). Em ambos os casos, a qualidade dos parâmetros estimados sofre influência, tanto do número de medições quanto da variância e covariância dos dados.

A confiabilidade dos parâmetros pode ser verificada através da análise da região de confiança. A região de confiança é a região do espaço de variáveis que concentra uma probabilidade $\mathbf{p}$ das possíveis flutuações observáveis do problema (Schwaab e Pinto, 2007). A aproximação elíptica da região de confiança, rigorosamente válida somente quando os erros experimentais possuem distribuição normal e o modelo é linear, é muito usada devido a sua simplicidade, pois requer apenas informações sobre o ponto ótimo estimado e a matriz de 
covariância (Schwaab et al., 2008). Porém, quando o caso avaliado difere dessas condições ideais, o que ocorre em muitos casos (Larentis et al., 2003), a forma elíptica fornece apenas uma aproximação da região de confiança dos parâmetros. Assim é necessário o emprego de métodos que não restrinjam a forma da região de confiança para uma melhor avaliação da confiabilidade dos valores estimados.

O objetivo principal deste trabalho é a avaliação do efeito tanto da quantidade de variáveis medidas quanto da correlação entre as variáveis medidas sobre a qualidade das estimativas dos parâmetros cinéticos de uma reação em série do tipo $\mathrm{A} \rightarrow \mathrm{B} \rightarrow \mathrm{C}$.

\section{METODOLOGIA}

Foram utilizados dados experimentais simulados empregando-se modelos cinéticos para um sistema de reações em série. Assumindo que as taxas das duas etapas da reação $\mathrm{R}$ são irreversíveis e de primeira ordem e que a reação é conduzida em um reator do tipo batelada, a variação da concentração dos compostos $\mathrm{A}, \mathrm{B}$ e $\mathrm{C}$ no tempo (t) é descrita pelo sistema de equações diferenciais apresentados na Equação 1. Considerando no tempo zero o reator contém apenas o reagente $\mathrm{A}$ com concentração $C_{A 0}$. Este sistema possuiu solução analítica, sendo que as concentrações de A, B e C são funções do tempo de reação conforme Equação 2.

$$
\begin{aligned}
& A \stackrel{k_{1}}{\longrightarrow} B \stackrel{k_{2}}{\longrightarrow} C \\
& \left\{\begin{array}{l}
\frac{d C_{A}}{d t}=-k_{1} C_{A} \\
\frac{d C_{B}}{d t}=k_{1} C_{A}-k_{2} C_{B} \\
\frac{d C_{C}}{d t}=k_{2} C_{B} \\
C_{A}=C_{A 0} \exp \left(-k_{1} t\right) \\
C_{B}=C_{A 0} \frac{k_{1}\left[\exp \left(-k_{1} t\right)-\exp \left(-k_{2} t\right)\right]}{k_{2}-k_{1}} \\
C_{C}=C_{A 0}\left\{1-\frac{\left[k_{2} \exp \left(-k_{1} t\right)-k_{1} \exp \left(-k_{2} t\right)\right]}{k_{2}-k_{1}}\right.
\end{array}\right\}
\end{aligned}
$$

Os dados experimentais foram simulados assumindo $k_{1}$ e $k_{2}$ iguais a 0,2 e 0,1 , respectivamente, e $C_{A 0}$ igual a 1 mol. $\mathrm{L}^{-1}$. Foi adicionado um erro aleatório, com distribuição normal e desvio padrão de 0,05 ao valor calculado para todas as concentrações de compostos, de forma a simular o efeito de erros experimentais. Para a estimação dos parâmetros cinéticos foram consideradas seis casos quanto à quantidade de variáveis observadas experimentalmente, como apresentado na Tabela 1. 
Tabela 1 - Casos considerados para a estimação de parâmetros cinéticos

\begin{tabular}{cc}
\hline Caso 1 & Têm-se a medida apenas da concentração do composto B \\
\hline Caso 2 & Têm-se a medida apenas da concentração do composto C \\
\hline Caso 3 & Têm-se a medida da concentração dos compostos A e B \\
\hline Caso 4 & Têm-se a medida da concentração dos compostos A e C \\
\hline Caso 5 & Têm-se a medida da concentração dos compostos B e C \\
\hline Caso 6 & Têm-se a medida da concentração de todos os compostos (A, B e C) \\
\hline
\end{tabular}

O método da máxima verossimilhança está baseado em hipóteses relativamente simples e permite a análise de virtualmente qualquer problema experimental de forma rigorosa, desde que se conheça de forma apropriada como se comportam os erros de medição na região experimental (Schwaab e Pinto, 2007). A primeira hipótese é que os erros experimentais seguem uma distribuição conhecida na região experimental. A segunda considera o modelo perfeito, ou seja, o modelo é perfeito e capaz de descrever exatamente as relações existentes entre as variáveis do problema e que os desvios são devidos exclusivamente a erros experimentais. Pressupõe-se, ainda, que o experimento é bem feito, sem a presença de erros grosseiros ou outliers. Assumindo que as hipóteses são verdadeiras e que as flutuações experimentais seguem a distribuição normal, a função a ser minimizada na estimação dos parâmetros do modelo (função objetivo) é conhecida como função de mínimos quadrados generalizados, Equação 3, pois leva em consideração tanto a variância das medidas como a covariância (ou correlação) entre elas.

$$
F_{o b j}=\sum_{i=1}^{N E}\left[\mathbf{y}_{i}^{e}-\mathbf{y}_{i}^{m}\left(\mathbf{x}_{i}^{e}, \boldsymbol{\theta}\right)\right]^{T} \mathbf{V}_{\mathbf{y}, i}^{-1}\left[\mathbf{y}_{i}^{e}-\mathbf{y}_{i}^{m}\left(\mathbf{x}_{i}^{e}, \boldsymbol{\theta}\right)\right]
$$

A região de confiança elíptica dos parâmetros pode ser representada pela Equação 4 (Schwaab e Pinto, 2007). Uma maneira alternativa de encontrar a região de confiança sem restringir sua forma, conhecida como Região de Confiança de Verossimilhança (Schwaab et al., 2008), é representada pela Equação 5.

$$
\begin{aligned}
& (\boldsymbol{\theta}-\hat{\boldsymbol{\theta}})^{T} \mathbf{V}_{\boldsymbol{\theta}}^{-1}(\boldsymbol{\theta}-\hat{\boldsymbol{\theta}}) \leq F_{o b j}(\hat{\boldsymbol{\theta}}) \frac{N P}{N-N P} F_{N P, N-N P}^{\alpha} \\
& F_{o b j}(\boldsymbol{\theta}) \leq F_{o b j}(\hat{\boldsymbol{\theta}})\left(1+\frac{N P}{N-N P} F_{N P, N-N P}^{\alpha}\right)
\end{aligned}
$$

A estimação foi realizada através da minimização da função objetivo com o uso de um algoritmo híbrido de otimização composto por um método estocástico acoplado a um método determinístico, em que a melhor solução encontrada pelo método do Enxame de Partículas (Kennedy e Eberhart, 1995) era utilizada como estimativa inicial para um método do tipo Gauss-Newton (Noronha et al., 1993). A utilização do método do Enxame de Partículas, apesar de ser computacionalmente intensivo para este problema com apenas 2 parâmetros para serem estimados, permite uma fácil obtenção da Região de Confiança de Verossimilhança (Schwaab et al., 2008). 


\section{RESULTADOS E DISCUSSÃO}

\subsection{Influência da quantidade de variáveis medidas}

Primeiramente foram estimados os parâmetros cinéticos $k_{1}$ e $k_{2}$, utilizando os dados experimentais simulados, considerando a variância experimental igual a 0,0025 , idêntica para todas as variáveis e experimentos. Os ajustes obtidos nos casos com diferentes quantidades de variáveis medidas para as concentrações dos compostos A, B e C, podem ser observados na Figura 1. Verifica-se que medindo apenas o produto final, caso 2, o modelo não consegue predizer com qualidade as concentrações dos outros dois compostos, A e B. Para os demais casos a capacidade preditiva do modelo foi idêntica.
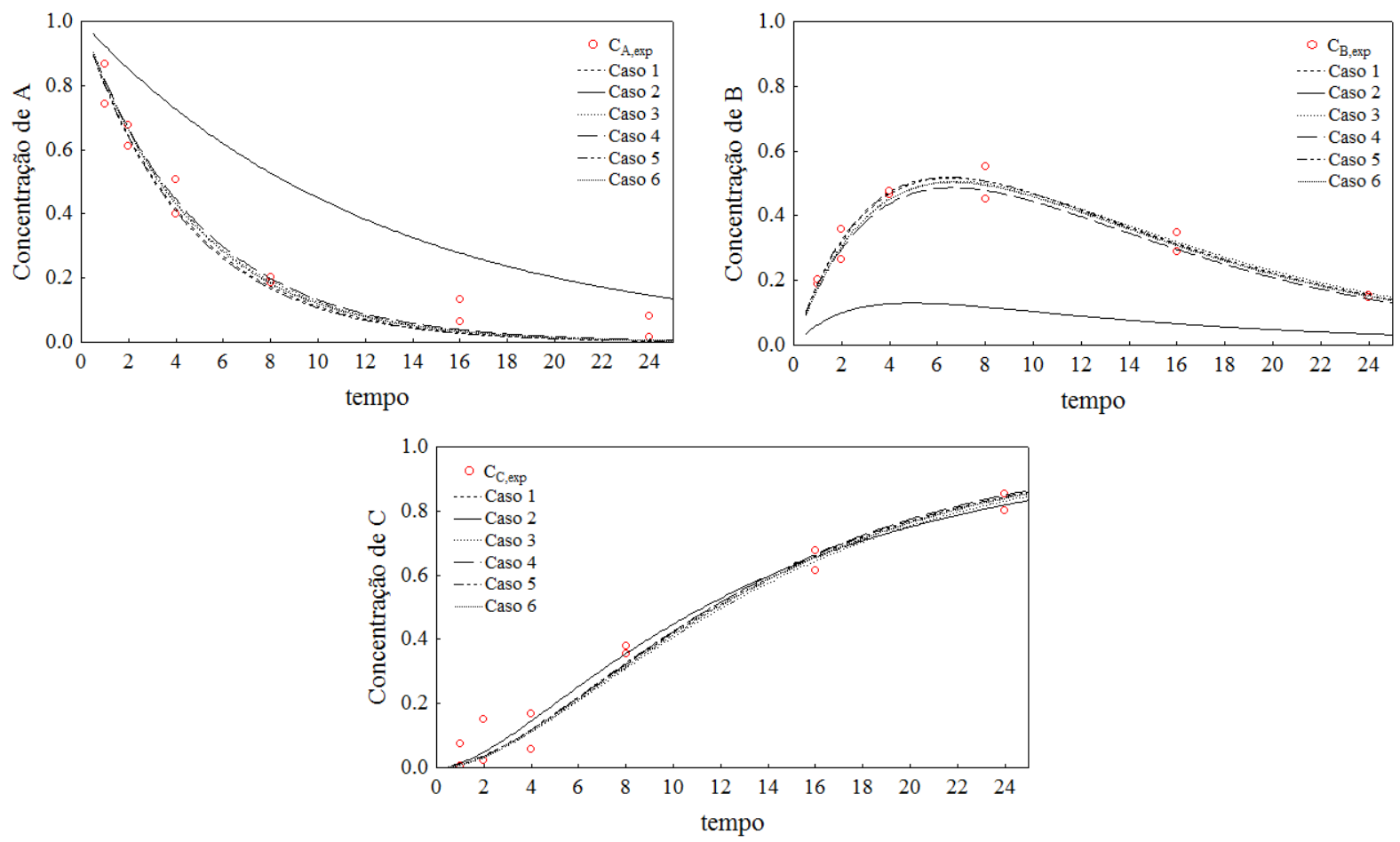

Figura 1 - Dados experimentais simulados e ajustes do modelo considerando os casos com diferentes números de variáveis medidas (concentrações dos compostos $\mathrm{A}, \mathrm{B}$ e $\mathrm{C}$ ).

Quanto aos valores dos parâmetros estimados, Tabela 2, verifica-se que há diferenças na precisão dos parâmetros cinéticos obtidos para os diferentes casos. Observa-se que os valores dos desvios padrão dos parâmetros mudam consideravelmente quando diferentes variáveis são medidas. Constatou-se que para a reação em série $\mathrm{A} \rightarrow \mathrm{B} \rightarrow \mathrm{C}$, medindo-se apenas as concentrações do reagente e do produto intermediário, é possível obter os valores das constantes cinéticas $k_{1}$ e $k_{2}$ com confiabilidade equivalente àquela que seria obtida caso as concentrações de todos os compostos fossem medidas. É importante ressaltar que esse resultado só se aplica quando não há correlação entre as variáveis medidas. 
Tabela 2 - Função objetivo, parâmetros cinéticos e desvio padrão para os seis casos

\begin{tabular}{|c|c|c|c|c|c|}
\hline Caso & $F_{o b j}$ & $k_{1}\left[\min ^{-1}\right]$ & $\sigma_{k 1}\left[\min ^{-1}\right]$ & $k_{2}\left[\min ^{-1}\right]$ & $\sigma_{k 2}\left[\min ^{-1}\right]$ \\
\hline 1 - Medindo $C_{B}$ & 4,67 & 0,2195 & 0,0120 & 0,1008 & 0,0049 \\
\hline 2 - Medindo $\mathrm{C}_{\mathrm{C}}$ & 10,92 & 0,0801 & 0,0112 & 0,4142 & 0,1872 \\
\hline 3 - Medindo $C_{A}$ e $C_{B}$ & 18,61 & 0,2073 & 0,0092 & 0,1003 & 0,0067 \\
\hline 4 - Medindo $C_{A}$ e $C_{C}$ & 26,36 & 0,2023 & 0,0132 & 0,1084 & 0,0098 \\
\hline 5 - Medindo $C_{B}$ e $C_{C}$ & 17,44 & 0,2239 & 0,0140 & 0,1015 & 0,0048 \\
\hline 6 - Medindo $C_{A}, C_{B}$ e $C_{C}$ & 31,96 & 0,2103 & 0,0093 & 0,1030 & 0,0052 \\
\hline
\end{tabular}

A qualidade dos parâmetros obtidos também pode ser verificada através das regiões de confiança, Figura 2. À exceção do caso 2, todas as hipóteses proporcionaram a obtenção de regiões de confiança de verossimilhança semelhantes às regiões elípticas, mostrando que, para estes casos, a aproximação elíptica pode ser aplicada com segurança na estimação dos parâmetros. Porém, para o caso em que apenas a concentração do produto final é medida (Caso 2) a região de confiança apresenta forma muito distante da elíptica. Este resultado está associado a pior qualidade do ajuste, observada na Figura 1. Indicando que a confiabilidade da aproximação elíptica da região de confiança dos parâmetros está associada à qualidade do ajuste dos dados experimentais, quanto melhor o ajuste, mais a região de confiança se aproxima da forma elíptica. Assim, pode-se afirmar que medindo apenas a concentração do produto final não é possível a obtenção de parâmetros cinéticos confiáveis para uma reação em série do tipo $\mathrm{A} \rightarrow \mathrm{B} \rightarrow \mathrm{C}$.
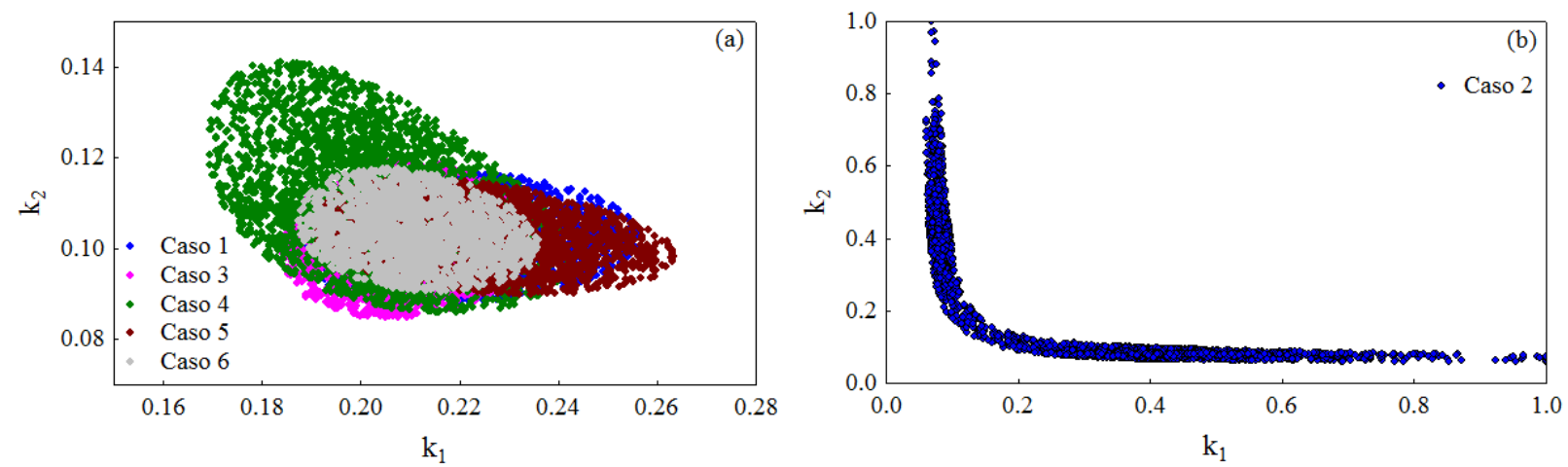

Figura 2 - Regiões de confiança dos parâmetros cinéticos para os Casos (a) 1, 3-6 (b) 2.

\subsection{Influência da correlação entre as medidas experimentais}

Para a avaliação da influência da correlação entre as variáveis sobre a qualidade dos parâmetros cinéticos estimados foram considerados apenas os casos em que duas concentrações eram medidas (Casos 3, 4 e 5) e o coeficiente de correlação foi variado de $-0,99$ a $+0,99$. Considerando que apenas as concentrações de A e B são medidas, Figura 3, observa-se que o aumento do coeficiente de correlação resulta no aumento do tamanho da 
região de confiança de verossimilhança e na piora da aproximação elíptica para a região de confiança. Estes resultados impactam na redução da confiabilidade dos parâmetros cinéticos obtidos.
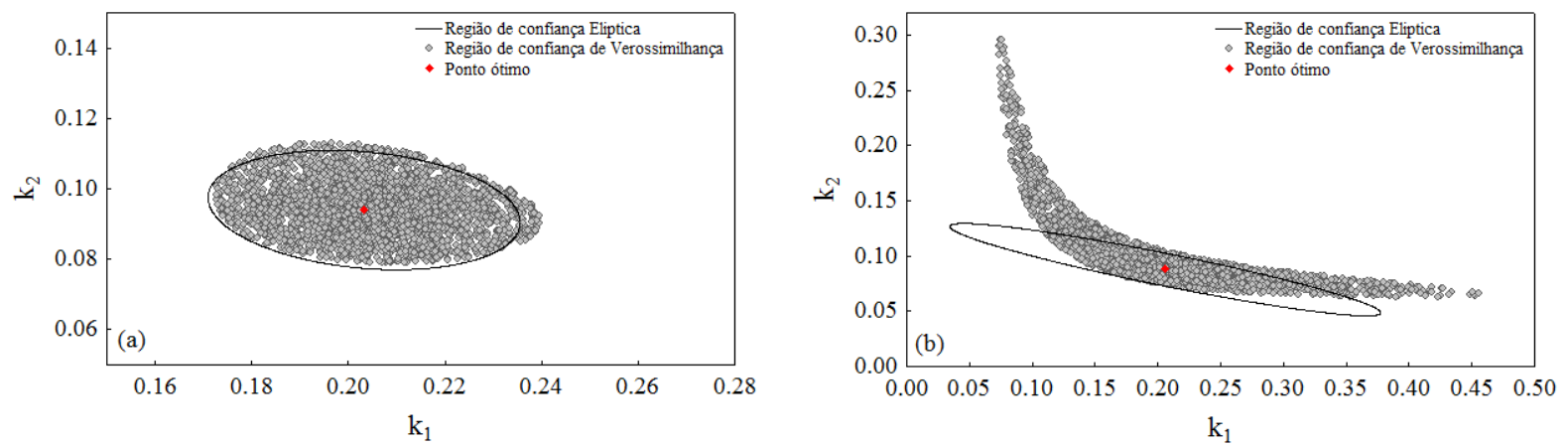

Figura 3 - Regiões de confiança elíptica e de verossimilhança para a hipótese de medirem-se as concentrações de A e B (Caso 3) com coeficiente de correlação de (a) -0,50 e (b) -0,99.

Comparando a Figura 3 b com a região de confiança do caso 1 na Figura 2a, fica claro que mesmo medindo dois compostos, se a correlação entre as medidas é alta, a precisão dos parâmetros é pior do que somente medindo a concentração do composto intermediário.

Para o caso em que as concentrações de A e C (Caso 4) são medidas, os resultados obtidos, variando-se o coeficiente de correlação entre as variáveis, são apresentados na Tabela 3. Pode-se verificar que o aumento do coeficiente de correlação entre $-0,99$ e $+0,99$ ocasionou um aumento do valor estimado para $k_{l}$ e uma redução do valor de $k_{2}$, assim como um aumento do desvio padrão do parâmetro $k_{1}$, sendo que o desvio padrão do parâmetro $k_{2}$ passa por um máximo.

Tabela 3 - Função objetivo, parâmetros cinéticos e desvio padrão para o Caso 4, medindo-se as concentrações de $\mathrm{A}$ e $\mathrm{C}$, com diferentes coeficientes de correlação.

\begin{tabular}{cccccc}
\hline Correlação & $F_{o b j}$ & $k_{1}\left[\mathrm{~min}^{-1}\right]$ & $\sigma_{k 1}\left[\mathrm{~min}^{-1}\right]$ & $k_{2}\left[\mathrm{~min}^{-1}\right]$ & $\sigma_{k 2}\left[\mathrm{~min}^{-1}\right]$ \\
\hline$\rho=-0,99$ & 277,24 & 0,1929 & 0,0083 & 0,1224 & 0,0048 \\
$\rho=-0,90$ & 39,86 & 0,1930 & 0,0089 & 0,1215 & 0,0055 \\
$\rho=-0,50$ & 21,90 & 0,1954 & 0,0109 & 0,1168 & 0,0079 \\
$\rho=0$ & 26,36 & 0,2023 & 0,0132 & 0,1084 & 0,0098 \\
$\rho=+0,50$ & 44,53 & 0,2161 & 0,0158 & 0,0953 & 0,0103 \\
$\rho=+0,90$ & 180,13 & 0,2349 & 0,0177 & 0,0817 & 0,0090 \\
$\rho=+0,99$ & 1681,13 & 0,2402 & 0,0180 & 0,0785 & 0,0086 \\
\hline
\end{tabular}

Quando foi considerado que as concentrações dos compostos B e C (Caso 5) eram medidas, observou-se tanto o deslocamento quanto a mudança da forma da região de confiança dos parâmetros com a variação do coeficiente de correlação, Figura 4. Nesse caso, fica evidente a redução da confiabilidade dos parâmetros, pois a região de confiança de 
verossimilhança aumenta consideravelmente, devido à correlação entre as variáveis medidas.

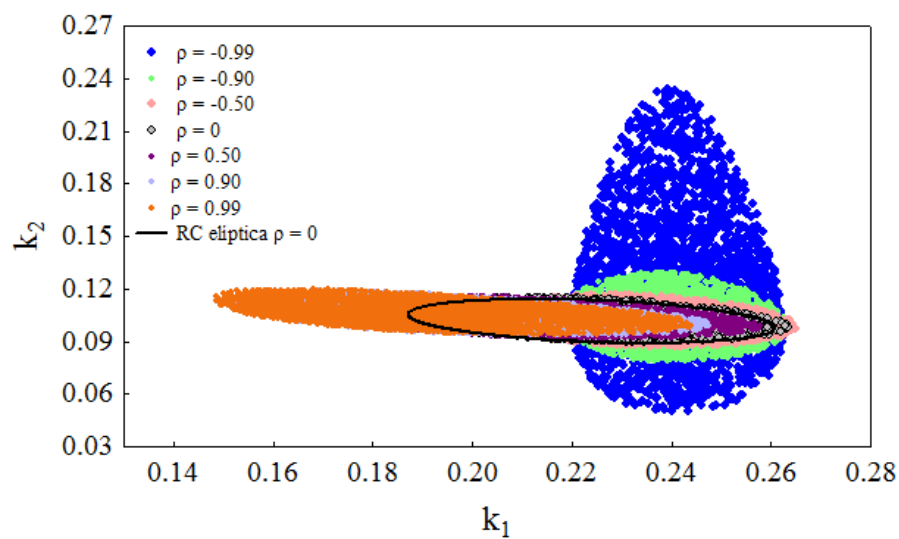

Figura 4 - Regiões de confiança para a hipótese de medirem-se as concentrações de B e C (Caso 5) com diferentes coeficientes de correlação.

\section{CONCLUSÃO}

Através da estimação de parâmetros cinéticos utilizando dados experimentais simulados para uma reação em série do tipo $\mathrm{A} \rightarrow \mathrm{B} \rightarrow \mathrm{C}$, verificou-se a influência da quantidade de variáveis medidas experimentalmente sobre a qualidade dos resultados obtidos. Constatou-se que com as medidas das concentrações do reagente e do composto intermediário é possível a estimação dos parâmetros cinéticos com qualidade, desde que estas medidas experimentais não estejam correlacionadas. A correlação entre as variáveis impacta diretamente na precisão dos valores estimados, tanto deslocando quanto alterando a forma das regiões de confiança dos parâmetros.

\section{AGRADECIMENTOS}

Os autores agradecem à Fundação de Amparo à Pesquisa do Estado do Rio Grande do Sul (FAPERGS) pela concessão de bolsa de estudo.

\section{NOMENCLATURA}

$\begin{array}{llcl}C_{j} & \text { Concentração do composto j } & \mathbf{V}_{\mathbf{y}, i} & \text { Matriz de covariância experimental } \\ F_{N P, N-N P}^{\alpha} & \text { Função de Fischer } & \mathbf{V}_{\boldsymbol{\theta}} & \text { Matriz de covariância dos parâmetros } \\ F_{o b j} & \text { Função objetivo } & \mathbf{x}_{i} & \text { Variável independente do experimento } \\ G L & \text { Graus de liberdade } & \mathbf{y}_{j}^{e} & \begin{array}{l}\text { Variável dependente j do experimento } \\ \text { i experimental }\end{array} \\ k_{R} & \text { Constante cinética da reação } R & \mathbf{y}_{i}^{m} & \text { Variável dependente j do experimento } \\ N & \text { Número total de medições } & \sigma & \text { Desvio padrão } \\ N E & \text { Número de medições no tempo } & \rho & \text { Coeficiente de correlação } \\ N P & \text { Número de parâmetros } & \hat{\boldsymbol{\theta}} & \text { Vetor de parâmetros ótimos }\end{array}$


$\boldsymbol{\theta} \quad$ Vetor de parâmetros

\section{REFERÊNCIAS}

BOX, G. E. P.; DRAPER, N. R. The Bayesian estimation of common parameters from several responses. Biometrika, v. 52, p. 355-365, 1965.

KENNEDY, J.; EBERHART, R. Particle swarm optimization. In: Proceedings of the IEEE International Conference on Neural Networks, Perth, Australia, v. 4, p. 1942-1948, 1995.

LARENTIS, A. L.; BENTES, Jr., A. M. P.; RESENDE, N. S.; SALIM, V. M. M.; PINTO, J. C. Analysis of experimental errors in catalytic tests for production of synthesis gas. Appl Catal A-Gen, v. 242, p. 365-379, 2003.

NORONHA, F. B.; PINTO, J. C.; MONTEIRO, J. L.; LOBÃO, M. W.; SANTOS, T. J.; 1993, Um Pacote Computacional para Estimação de Parâmetros e Projeto de Experimentos, Relatório Técnico PEQ/COPPE, Universidade Federal do Rio de Janeiro, Rio de Janeiro, RJ, Brasil.

SANTOS, T. J. PINTO, J. C. Taking variable correlation into consideration during parameter estimation. Braz J Chem Eng, v. 15, p. 1-20, 1998.

SCHWAAB, M.; PINTO, J. C. Análise de Dados Experimentais I - Fundamentos de Estatística e Estimação de Parâmetros. E-papers, Rio de Janeiro, 2007.

SCHWAAB, M.; BISCAIA, Jr, E. C.; MONTEIRO, J. L.; PINTO, J. C. Nonlinear parameter estimation through particle swarm optimization. Chem Eng Sci, v. 63, p. 1542-1552, 2008. 